Classification

Physics Abstracts 6.700

\title{
ÉTUDE THÉORIQUE DE LA RÉGION CATHODIQUE DANS UN ARC A CATHODE LIQUIDE
}

\author{
G. THIELL et J. C. BRAUN \\ Laboratoire de Physique des Milieux Ionisés \\ Université de Nancy I, C. O. 140, 54037 Nancy Cedex, France \\ (Reçu le 4 juillet 1975, révisé le 23 février 1976, accepté le 18 mai 1976)
}

\begin{abstract}
Résumé. - Nous développons un modèle de gaine cathodique dans un arc généré à partir d'une cathode liquide en potassium, dans un gaz inerte (argon) à une pression de quelques torrs ; à partir d'un bilan général des échanges existants au voisinage de la cathode, nous avons construit les diagrammes d'existence des spots constituant la tache cathodique ; nous avons envisagé des intensités de décharge allant jusqu'à $100 \mathrm{~A}$; nous avons également abordé le problème de la part de l'émission thermoélectronique par rapport à celle de l'émission de champ dans la formation d'électrons à la cathode.
\end{abstract}

Abstract. - We analyse a cathode sheath model in an arc generated from a potassium liquid cathode in an inert gas (Argon) at a pressure of a few torrs ; we have used a complete balance of the exchanges near the cathode ; we have then drawn the existence-diagrams of the spots that make up the cathode area considering arc intensities from 0 to $100 \mathrm{~A}$. We have also considered the ratio of thermal electron emission to the field emission from the cathode surface.

Introduction. - On sait que le phénomène de tache cathodique dans un arc électrique est étroitement lié au mode d'émission électronique à la cathode. On considère généralement que les cathodes constituées par des métaux ayant un point de fusion élevé (W, Mo,...) émettent des électrons par effet thermoionique et que celles qui présentent une faible température de fusion $(\mathrm{Cu}, \mathrm{Hg}, \ldots)$ émettent des électrons par effet de champ.

La nature du métal utilisé comme cathode est déterminante pour les propriétés du plasma d'arc. Dans le cas d'une cathode liquide, c'est celle-ci qui fournit les ions du plasma alors que dans les décharges entre électrodes solides, c'est habituellement un gaz injecté qui est ionisé par un processus physique. Les métaux alcalins présentent des propriétés intéressantes par rapport aux autres métaux : faible travail de sortie, faible potentiel d'ionisation, taux d'évaporation élevé. Ceci autorise à espérer des densités électroniques et ioniques élevées dans la zone cathodique comme le confirment les résultats de cette étude.

Le plasma créé dépend fortement des processus ayant lieu dans la région cathodique ; pour tenter d'expliquer ces derniers, nous développons un modèle de gaine permettant de tracer le diagramme d'existence de l'arc à cathode en potassium, c'est-à-dire la région du plan $T(j)$ où nous choisissons $T$ la température de la tache cathodique en fonction de la densité de courant total $j$ qui la traverse. Cette zone est déterminée par trois conditions de base : la conservation de la densité de courant, la conservation des neutres et la conservation de l'énergie. G. Ecker [1] a défini cette méthode et l'a appliquée au cas d'un arc à cathode en cuivre notamment, en calculant le champ électrique cathodique à partir de l'équation de Mac Keown. Dans la référence [2], il propose un modèle de gaine cathodique que nous allons adapter au cas d'une électrode à l'état liquide et sur les bases duquel nous construisons un nouveau diagramme d'existence. Dans notre modèle, nous écrivons le bilan d'énergie à la surface du liquide cathodique et nous tenons compte des électrons qui, thermalisés dans le plasma d'arc, diffusent vers la cathode et que nous appellerons pour cette raison électrons contre-diffusés.

1. Equations de base décrivant le modèle de gaine cathodique. - Le modèle de gaine que nous développons repose sur plusieurs hypothèses fondamentales :

1) Nous distinguons devant la surface cathodique une région de charge d'espace et une région d'ionisation globalement neutre.

2) Dans la région de charge d'espace, nous admettons qu'il n'y a ni ionisation, ni recombinaison et que les densités de courant électronique et ionique se conservent. 
3) Dans la région d'ionisation, nous supposons que les densités électronique et ionique sont assez élevées pour que dans cette zone l'équilibre thermodynamique local (E. T. L.) soit réalisé.

4) Les électrons sont émis par la cathode à la température $T$ du spot ; la forte densité de vapeur devant la tache cathodique et le fait que les ions sont créés dans la région d'ionisation définie par notre modèle, nous font dire que les températures des ions et des atomes neutres provenant de la tache cathodique sont égales entre elles et égales à la température $T$ de cette tache.

5) Du point de vue mathématique, une description unidimensionnelle de la région de charge d'espace est possible, étant donné la valeur élevée du champ électrique cathodique qui imprime aux charges électriques une vitesse perpendiculaire à la surface de la cathode.

La densité de courant total dans la tache cathodique s'écrit :

$$
j=j_{\mathrm{e}}+j_{+}
$$

si l'on néglige la densité de courant $j_{\mathrm{D}}$ due aux électrons contre-diffusés. La densité de courant électronique $j_{\mathrm{e}}$ émise par le métal cathodique est donnée par la relation [3] :

$$
j_{\mathrm{e}}\left(T, E_{\mathrm{c}}, \varepsilon\right)=e \int_{-W_{a}}^{+\infty} D\left(E_{\mathrm{c}}, W\right) \cdot N(T, \varepsilon, W) \mathrm{d} W
$$

où $T, \varepsilon, W_{\mathrm{a}}$ et $e$ représentent respectivement la température de la tache cathodique, l'énergie de Fermi, le potentiel interne du métal cathodique et la charge de l'électron.

$D\left(E_{\mathrm{c}}, W\right)$ est la probabilité d'émission d'un électron d'énergie $W$ du métal soumis au champ électrique $E_{\mathrm{c}}$. $N(T, \varepsilon, W) \mathrm{d} W$ est le nombre d'électrons frappant la barrière de potentiel par seconde et par unité de surface et ayant une énergie comprise entre $W$ et $W+\mathrm{d} W$.

$j_{+}$est la densité de courant ionique collecté à la cathode. Son calcul exact est délicat. Sa valeur maximale peut être écrite sous la forme :

$$
j_{+}=\frac{e}{4} \sum_{v} v n_{+v}\left(\frac{3 k T}{m_{+}}\right)^{1 / 2}
$$

où $n_{+v}$ désigne la densité des ions $v$ fois ionisés, $k$ et $m_{+}$ étant respectivement la constante de Boltzmann et la masse d'un ion.

Une remarque s'impose. Le problème se pose de savoir ce que devient le travail de sortie $\varphi$ d'un métal à l'état liquide : il est étroitement lié à l'énergie de Fermi $\varepsilon$ :

$$
\varphi=-\varepsilon
$$

si l'on prend pour origine des énergies, l'énergie de l'électron libre en dehors du métal.

Dans un métal liquide où la densité électronique est élevée, les électrons ne sont pas distribués uniformément à travers le liquide mais forment un écran autour des ions positifs [4]. Les corrélations de Coulomb sont la principale cause d'interaction perturbant le gaz d'électrons. E. Daniel et S. H. Vosko [5] montrent que l'énergie de Fermi reste bien définie dans le cas d'un gaz d'électrons interagissant entre eux et, plus la densité électronique est élevée, plus la discontinuité de la distribution en énergies est abrupte pour l'énergie de Fermi.

Nous choisissons pour nos calculs théoriques, dans le cas du potassium liquide, le travail de sortie $\varphi=2 \mathrm{eV}$ du potassium à l'état solide.

Dans la région d'ionisation, les électrons accélérés par la tension cathodique sont thermalisés à la température $T_{-}$par collisions ionisantes. Pour un tel plasma en E. T. L., le coefficient d'ionisation est donné par l'équation de Saha que nous écrivons sous la forme :

$$
\begin{array}{r}
\frac{n_{+(v+1)} \cdot n_{-}}{n_{+v}}=2 \frac{g_{v+1}}{g_{v}}\left[\frac{2 \pi m_{-} k T_{-}}{h^{2}}\right]^{3 / 2} \times \\
\times \exp \left(-\frac{e U_{i(v+1)}}{k T_{-}}\right)
\end{array}
$$

avec :

$$
n_{0}+\sum_{v} n_{+v}=\frac{P(T)}{k T}
$$

et :

$$
n_{-}=\sum_{v} v n_{+v}
$$

où $n_{0}$ et $n_{-}$représentent respectivement la densité des atomes neutres et celle des électrons. $P(T)$ désigne la pression de vapeur au-dessus de la tache cathodique, $g_{v+1}$ et $g_{v}$ respectivement les poids statistiques des ions $(v+1)$ et $v$ fois ionisés, $U_{i(v+1)}$ le potentiel d'ionisation d'ordre $(v+1), h$ et $m_{-}$respectivement la constante de Planck et la masse de l'électron.

Le champ électrique $E_{\mathrm{c}}$ à la surface de la cathode est solution de l'équation de Poisson que nous pouvons écrire dans la région de charge d'espace sous la forme :

$$
\frac{\mathrm{d}^{2} U}{\mathrm{~d} x^{2}}=-\frac{e}{\varepsilon_{0}}\left(n_{+}-n_{-}-n_{\mathrm{e}}\right)
$$

avec :

$$
\begin{aligned}
& n_{+}=\frac{\sum_{v} v n_{+v}^{0}}{\left[1-\frac{2 e v\left(U-U_{\mathrm{cr}}\right)}{k T}\right]^{1 / 2}} \\
& n_{\mathrm{e}}=\frac{j_{\mathrm{e}}}{e}\left(\frac{m_{-}}{k T}\right)^{1 / 2}\left[1-\frac{2 e\left(U-U_{\mathrm{cr}}\right)}{k T}\right]^{1 / 2}
\end{aligned}
$$

et :

$$
n_{-}=\int_{-}^{+\infty} \sqrt{-2 e\left(U_{k}-U\right) / m_{-}} f_{-}(x, v) \mathrm{d} v
$$

où $n_{+}$et $n_{\mathrm{e}}$ sont respectivement les densités des ions et des électrons émis par la cathode. L'indice 0 indique la valeur de ces paramètres au point $x=0$ (Fig. 1). 


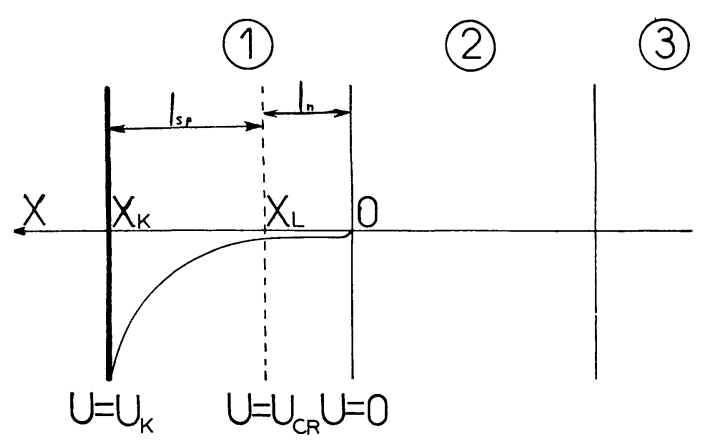

Fig. 1. - Modèle de gaine cathodique et forme du potentiel de gaine. (1) Gaine; (2) Région d'ionisation; (3) Plasma.

La fonction de distribution des électrons rétrodiffusés s'écrit :

$$
f_{-}(x, v)=\left(\frac{m_{-}}{2 \pi k T_{-}}\right)^{1 / 2} \exp \left[-\frac{m_{-} v^{2}}{2 k T_{-}}+\frac{e U}{k T_{-}}\right]
$$

soit :

$$
n_{-}=\frac{1}{2} n_{-}^{0} \exp \left(\frac{e U}{k T_{-}}\right)\left[1+\operatorname{erf}\left(\sqrt{\frac{e\left(U-U_{k}\right)}{k T_{-}}}\right)\right] .
$$

L'expression de $E_{\mathrm{c}}$ peut alors s'écrire sous la forme :

$$
\begin{array}{r}
E_{\mathrm{c}}^{2}=\frac{2 k}{\varepsilon_{0}} \sum_{v} v n_{+}^{0}\left[\left[T\left(1-\frac{2 e U_{\mathrm{c}}}{k T}\right)^{1 / 2}-1\right]-\right. \\
\left.-T_{-} v \exp \left(\frac{e U_{\mathrm{cr}}}{k T_{-}}\right)\right]
\end{array}
$$

avec $U_{\mathrm{c}}=U_{\mathrm{k}}-U_{\mathrm{cr}}, \varepsilon_{0}$ étant la permittivité du vide.

Dans ce calcul nous avons négligé la contribution des électrons émis par la cathode, ce qui se justifie numériquement.

Le calcul peut être complété et les phénomènes cathodiques mieux compris [2] si l'on considère que la région de charge d'espace peut être divisée en deux zones : une région quasi neutre $l_{n}$ et une zone de charge d'espace effective $l_{\text {sp }}$ (Fig. 1). Nous verrons que le champ électrique $E_{\mathrm{cr}}$ dans la gaine à la limite entre les régions quasi neutre et de charge d'espace est très faible. Le paramètre $l_{\mathrm{sp}}$ est défini par l'équation:

$$
l_{\mathrm{sp}}=\int_{0}^{U_{\mathrm{c}}} \frac{\mathrm{d} U}{E(U)}
$$

Cette relation impose la valeur de la chute de potentiel dans la région de charge d'espace effective de telle façon que la densité des électrons contre-diffusés dans cette zone soit négligeable.

La densité de ces électrons est alors insignifiante à la cathode $\left(x=x_{K}\right)$. Cependant, au point $x=x_{l}$, elle peut encore intervenir et elle est déterminée en ce point par la température $T_{-}$et le potentiel $U_{\text {cr. }}$ A priori, nous ne pouvons négliger l'effet des électrons contrediffusés dans le calcul du champ électrique $E_{\mathrm{c}}$ car le système $\left(E_{\mathrm{c}}, U_{\mathrm{c}}, T_{-}\right)$étant self-consistent, $E_{\mathrm{c}}$ dépend de l'état du milieu en $x=x_{l}$, ce que l'on constate bien sur les relations (4) et (7). Dans le second membre de (7), l'exponentielle qui représente la contribution des électrons rétrodiffusés au point $x=x_{K}$ est négligeable devant celle qui représente la contribution de ces électrons en $x=x_{l}$.

Il nous reste à calculer la chute cathodique de l'arc. Nous supposons dans la suite que les ions sont une seule fois ionisés afin de simplifier le calcul sans en restreindre la généralité. Compte tenu de la forte tension de vapeur dans la tache cathodique le mouvement des ions dans la région de charge d'espace effective est régi par l'équation :

$$
j_{+}=n_{+} e \mu_{+} \frac{\mathrm{d} U}{\mathrm{~d} x}
$$

où $\mu_{+}$représente la mobilité des ions.

En intégrant l'équation de Poisson dans cette région, nous trouvons la loi bien connue :

$$
U_{\mathrm{c}}=\frac{2}{3}\left(\frac{2 j_{+}}{\varepsilon_{0} \mu_{+}}\right)^{1 / 2} l_{\mathrm{sp}}^{3 / 2}
$$

et afin de tenir compte des électrons contre-diffusés nous utilisons le principe de sélection par le potentiel minimum (principe de Steenbeck) tel qu'il a été défini dans [2] :

$$
U_{\mathrm{c}}=\frac{k T_{-}}{e} \ln \left(\frac{3 e U_{\mathrm{i}}}{2 k T_{-} \sqrt{f}}\right)
$$

où $f$ est le facteur de perte d'énergie électronique.

Il est à noter qu'à partir de l'équation (9), on retrouve la même expression de $U_{c}$ que celle qu'on déduit d'une décharge à faible pression.

2. Diagramme d'existence. - G. Ecker détermine les limites de $j_{+}$dans les cas suivants :

En première approximation, on peut dire que l'énergie $j_{\mathrm{e}} U_{\mathrm{c}}$ acquise par les électrons accélérés dans la chute cathodique $U_{\mathrm{c}}$ et entrant dans la région d'ionisation doit au moins être égale à l'énergie d'ionisation $j_{+} U_{\mathrm{i}}$; soit :

$$
j_{+} U_{\mathrm{i}} \leqslant j_{\mathrm{e}} U_{\mathrm{c}} .
$$

Notons que les pertes par diffusion et les chocs inélastiques sont négligés.

A cause des processus d'ionisation et de recombinaison entre le plasma (pl) où électrons et ions sont thermalisés respectivement aux températures $T_{-}$et $T_{+}$ et la région de charge d'espace (sp), nous avons les relations :

$$
\left(\frac{j_{-}}{j_{+}}\right)_{\mathrm{pl}}=\frac{n_{-}}{n_{+}}\left(\frac{m_{+} T_{-}}{m_{-} T_{+}}\right)^{1 / 2}
$$

et

$$
\left(\frac{j_{\mathrm{e}}}{j_{+}}\right)_{\mathrm{sp}} \leqslant \alpha\left(\frac{m_{+} T_{-}}{m_{-} T_{+}}\right)^{1 / 2}
$$

$\alpha$ est un coefficient numérique voisin de 2 [6] et $m_{+}$ la masse d'un ion.

La relation (11) donne une limite supérieure de la densité de courant ionique et la relation (12) une limite inférieure dans le diagramme $T(j)$. 
Deux autres limites de $j_{+}$sont déterminées en supposant d'une part que tous les atomes neutres vaporisés dans la tache cathodique sont ionisés dans le plasma adjacent à la cathode et retournent à la cathode avec une distribution maxwellienne :

$$
j_{+} \leqslant \frac{e}{4}\left(\frac{P(T)}{m_{+} k T / 3}\right)^{1 / 2}
$$

et d'autre part qu'aucuns des atomes neutres vaporisés n'est ionisé. Ceci impose que le rayon du spot $a=(I / \pi j)^{1 / 2}$ soit beaucoup plus grand que le libre parcours moyen

$$
\lambda_{\mathrm{e}}=\frac{1}{n_{0} Q_{\mathrm{e}}}
$$

des électrons dans le gaz neutre :

$$
\left(\frac{I}{\pi j}\right)^{1 / 2} \gg \frac{1}{n_{0} Q_{\mathrm{e}}}
$$

où $Q_{\mathrm{e}}$ est la section efficace de collision électron-neutre. $n_{0}$ est la densité de particules évaporées et $I$ l'intensité du courant de décharge.

Ces deux relations déterminent encore deux courbes limites dans le diagramme $T(j)$.

Une dernière valeur limite de $j_{+}$nous est donnée par l'équation du bilan d'énergie à la surface de la cathode. Dans le cas particulier d'un arc à cathode en métal liquide nous pouvons préciser davantage le bilan général établi par G. Ecker dans la référence [1] grâce aux remarques suivantes. Des données expérimentales nous permettent de connaître de façon précise les conductivités électrique et thermique du potassium liquide entre le point de fusion et le point critique. Nous devons tenir compte de la forte évaporation au-dessus $\mathrm{du}$ liquide cathodique. Nous calculons l'énergie de conduction dans l'électrode directement à partir de la valeur $\left(\psi_{1} \frac{\mathrm{d} T}{\mathrm{~d} x}\right)_{\mathrm{c}}$ où $\psi_{1}$ est la conductivité thermique du liquide cathodique.

Faisons l'hypothèse que le gaz de remplissage de l'enceinte où se produit la décharge (argon) n'est pas ionisé dans le plasma. Ceci peut se justifier en partie par le fait que le potentiel d'ionisation de l'argon $(15,75 \mathrm{eV})$ est beaucoup plus élevé que la tension anode-cathode (de l'ordre de $8 \mathrm{~V}$ ) observée expérimentalement et que le potentiel d'ionisation du potassium $(4,34 \mathrm{eV})$,

Examinons d'abord les flux d'énergie traversant la surface cathodique dans le sens plasma-cathode :

a) $F_{1}=j_{+} U_{\mathrm{c}}$ est dû aux ions bombardant la cathode,

b) $F_{2}=j_{+}\left(U_{\mathrm{i}}-\varphi\right) a_{+}$est l'énergie de neutralisation des ions, $\varphi$ étant le travail de sortie du liquide cathodique et $a_{+}$un coefficient d'accommodation $(\leqslant 1 / 2)[7]$,

c) $F_{3}=\frac{0,32}{\sqrt{\pi}} j^{3 / 2} I^{1 / 2} \rho[8]$ est la densité d'énergie due à l'effet Joule, $\rho$ étant la résistivité du liquide cathodique.

Le calcul de $\rho$ se fait à partir de la loi expérimentale bien vérifiée pour les métaux liquides et notamment les alcalins, depuis le point de fusion $T_{\mathrm{F}}$ jusqu'au point critique $T_{\mathrm{c}}$

$$
\left[\frac{\rho\left(T_{\mathrm{F}}\right)}{\rho(T)}+b\right]\left[\frac{T_{-} T_{\mathrm{F}}}{T_{\mathrm{c}}-T_{\mathrm{F}}}+b\right]=a
$$

où $a$ et $b$ sont deux constantes [9].

En effet, pour les métaux liquides, soumis aux conditions physiques extrêmes régnant dans un spot cathodique, les conductivités électrique et thermique tendent rapidement vers zéro ce que ne permet pas de prévoir la loi Bloch-Grüneisen.

Examinons maintenant les flux d'énergie traversant la surface cathodique dans le sens cathode-plasma.

a) $E_{1}=W(T) \cdot Q(T)$ est la perte d'énergie par évaporation. $Q(T)$ est la chaleur de vaporisation du potassium liquide donné par Kelley [10] sous la forme :

$$
Q(\text { cal/atg })=21788-2,73 \mathrm{~T} \text {. }
$$

Pour une cathode liquide, le coefficient d'évaporation $W$ des atomes du métal est très élevé. Dans le cas du potassium nous avons :

$$
\log W\left(\mathrm{~g} / \mathrm{s} \cdot \mathrm{cm}^{2}\right)=6,922-\frac{4503}{T}-\frac{1}{2} \log T .
$$

b) $E_{2}=j_{\mathrm{e}} \varphi$ est le refroidissement de la cathode par émission électronique.

c) $E_{3}=-\left(\psi_{1} \frac{\mathrm{d} T}{\mathrm{~d} x}\right)_{\mathrm{c}}$ est la perte d'énergie par conduction à travers l'électrode. Ce terme se calcule à partir de l'équation :

$$
\frac{\mathrm{d}}{\mathrm{d} x}\left(\psi_{1} \frac{\mathrm{d} T}{\mathrm{~d} x}\right)=-\rho j^{2}
$$

Le changement de variable

$$
y=\int_{0}^{x} \frac{\mathrm{d} x}{\psi_{1}}
$$

nous permet d'intégrer l'équation précédente dans le cas où $\psi_{1}$ dépend de $T$, soit :

$$
\left(\frac{\mathrm{d} T}{\mathrm{~d} y}\right)_{1}^{2}-\left(\frac{\mathrm{d} T}{\mathrm{~d} y}\right)_{c}^{2}=-\int_{T}^{T_{1}} 2 j^{2} \rho \psi_{1} \mathrm{~d} T
$$

avec la condition de stabilité $\mathrm{d} T / \mathrm{d} x=0$ pour $x=1$ qui correspond à la température $T_{1}$ constante du liquide cathodique.

En utilisant la loi de Wiedemann-Franz :

$$
\rho \psi_{1}=L_{0} T
$$

avec

$$
L_{0}=\frac{\pi^{2} k^{2}}{3 e^{2}}=2,443 \times 10^{-8} V^{2} \cdot \mathrm{K}^{-2}
$$


nous obtenons finalement :

$$
E_{3}=j\left[L_{0}\left(T^{2}-T_{1}^{2}\right)\right]^{1 / 2} .
$$

On ne considère ici que la conductivité thermique due aux électrons en négligeant celle du réseau.

Nous tenons implicitement compte de l'énergie absorbée ou dégagée par des réactions chimiques pouvant éventuellement se produire à la surface de l'électrode liquide dans la tache cathodique et de l'énergie de rayonnement dans le terme $F_{2}$. De ce fait le coefficient $a_{+}$est mal connu.

L'équation du bilan d'énergie est alors entièrement déterminée :

$$
\begin{aligned}
& W(T) \cdot Q(T)+j_{\mathrm{e}}\left(T, E_{\mathrm{c}}\right) \cdot \varphi+ \\
& \quad+j\left[L_{0}\left(T^{2}-T_{1}^{2}\right)\right]^{1 / 2}=j_{+}\left(T, E_{\mathrm{c}}\right) \cdot U_{\mathrm{c}} \\
& \quad+j_{+}\left(T, E_{\mathrm{c}}\right) \cdot\left(U_{\mathrm{i}}-\varphi\right) \cdot a_{+}+\frac{0,32}{\sqrt{\pi}} j^{3 / 2} I^{1 / 2} \rho(T) .
\end{aligned}
$$

3. Résolution numérique et discussion. - L'équation de Poisson (6) qui permet de déterminer le modèle de gaine de la figure 1 (avec $U=0$ pour $x=0$ ), a été résolue en fonction du rapport des températures $T_{-} / T$ et en tenant compte des électrons contre-diffusés (Fig. 2).

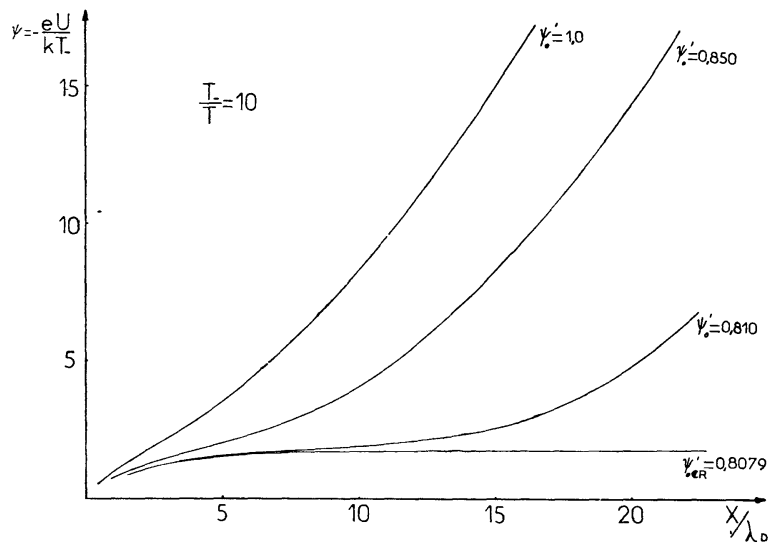

Fig. 2. - Distribution de potentiel dans la gaine cathodique. L'épaisseur de la gaine est comptée en longueur de Debye $\left(\lambda_{\mathrm{D}}\right)$. Le paramètre $\psi_{0}^{\prime}$ est le champ électrique réduit au point $x=0$.

Le paramètre $\psi_{0}^{\prime}$ détermine le champ électrique réduit au point $x=0$. Pour $T_{-} / T=10$, la valeur relativement élevée de $\psi_{\text {ocr }}^{\prime}$ (de l'ordre de $5 \times 10^{8} \mathrm{~V} / \mathrm{m}$ ) pour laquelle le potentiel est constant à travers toute la gaine, permet d'expliquer qu'au bout d'un parcours $l_{\text {n }}$ dans la région (1) (Fig. 1), la population des électrons contre-diffusés est négligeable devant la charge d'espace positive. Dans la zone de charge d'espace effective $l_{\mathrm{sp}}$, on peut donc ne tenir compte que des ions lors de la résolution de l'équation de Poisson donnant $E_{\mathrm{c}}$.

Bien que sensiblement au bout de 5 fois la longueur de Debye $\lambda_{D}$ le champ électrique soit pratiquement nul, le potentiel retardateur $U$ freine les électrons venus du plasma. Au point $x=x_{1}$, le champ électrique a une valeur négligeable par rapport à $E_{\mathrm{c}}$.

L'influence des électrons thermalisés qui diffusent vers la cathode se fait sentir sur la distribution de potentiel dans la gaine cathodique et nous en tenons effectivement compte dans l'équation (10).

Nous constatons sur la relation (7) que plus $U_{\text {cr }}$ est petit, plus les électrons thermalisés remonteront loin dans la gaine le potentiel retardateur et ceci d'autant plus que leur énergie $k T$ sera élevée.

Les équations (1), (2), l'équation de Mac Keown et l'équation (11) forment un système qui nous permet de calculer $T$ en fonction de $j$. Dans le cas d'une cathode en potassium liquide, nous nous fixons la valeur de la chute cathodique $\left(U_{\mathrm{c}}=6 \mathrm{~V}\right.$ est la valeur minimale de la tension anode-cathode observée expérimentalement). Nous appelons $T_{1}$ la courbe ainsi obtenue dans le plan $T(j)$ (Fig. 3).

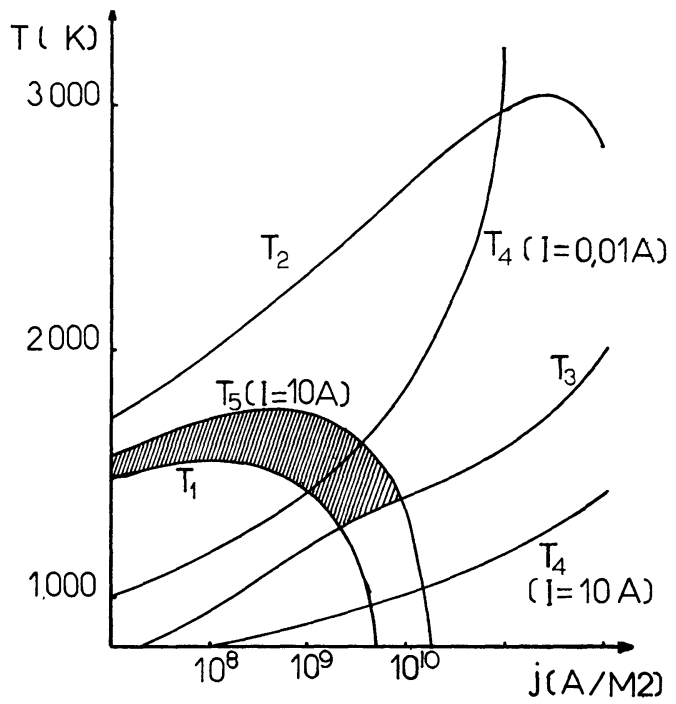

FIG. 3. - Diagramme d'existence de l'arc à cathode en potassium liquide obtenu en calculant $E_{\mathrm{c}}$ à l'aide de l'équation de Mac Keown.

Si nous résolvons le système précédent en remplaçant successivement l'équation (11) par l'équation (12), puis (13), (14) et (15), nous déterminons respectivement les courbes $T_{2}, T_{3}, T_{4}$ et $T_{5}$ dans le plan $T(j)$ (Fig. 3). La partie hachurée ou diagramme d'existence est le domaine du plan $T(j)$ où toutes les inéquations (11) à (15) sont vérifiées simultanément.

Considérons maintenant le système formé par les équations (1), (2), (3), (4), (5), (6) et (10) avec $v=1$. Les inconnues de ce système sont la température électronique $T_{-}$, la chute cathodique $U_{\mathrm{c}}$, le champ électrique $E_{\mathrm{c}}$ à la surface de la cathode, la densité ionique $n_{+}^{0}$, les densités de courant $j_{+}, j_{\mathrm{e}}$ et $j$ et la température $T$ de la tache cathodique. Nous nous fixons un seul paramètre : l'intensité $I$ du courant de décharge qu'il est facile de contrôler expérimentalement. De plus, l'équation (8) donne l'épaisseur $l_{\mathrm{sp}}$ de la région de charge d'espace effective.

Pour que nous puissions calculer la température $T$ en fonction de la densité du courant total $j$, il faut soit déterminer une condition supplémentaire, soit se donner l'une des inconnues.

Sur la figure 4, nous avons porté le diagramme 
d'existence délimité par les courbes de la figure 3, ainsi que la température $T$ du spot en fonction de la densité pour $U_{\mathrm{c}}$ constant, $T_{-}$constant et $j_{+} / j$ constant. On constate sur cette figure que le rapport de température $T_{-} / T=10$ correspond bien à des points de la zone d'existence de l'arc.

A l'aide du modèle de gaine cathodique développé dans ce travail, nous traçons le nouveau diagramme d'existence de l'arc à cathode en potassium liquide, à partir des équations (1) à (6) et l'équation (10) auxquelles nous ajoutons l'une des équations (11), (12), (13), (14) et (15) pour déterminer respectivement les courbes $T_{1}, T_{2}, T_{3}, T_{4}$ et $T_{5}$ de la figure 5 .

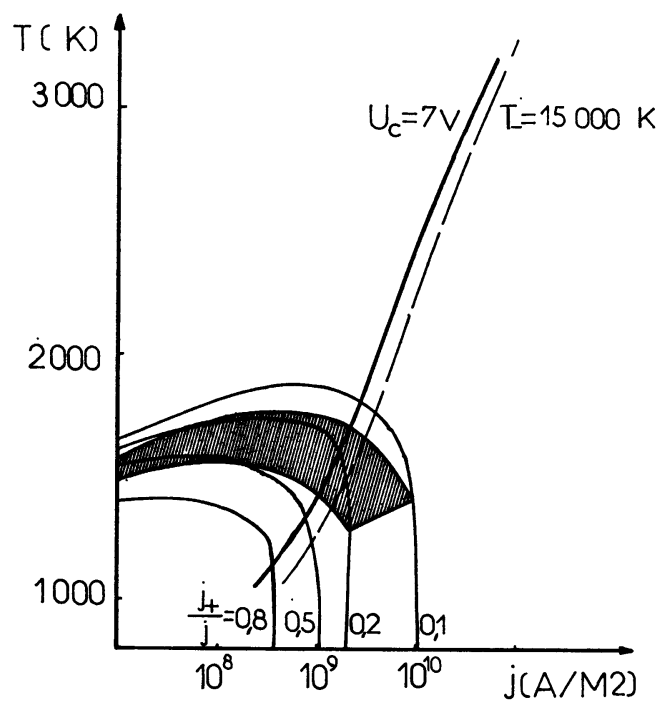

FIG. 4. - Chute cathodique $U_{\mathrm{c}}$. - - - - Température électronique $T_{-} \longrightarrow$ Rapport des courants ioniques et électroniques $j_{+} / j_{\mathrm{e}}$ en fonction de la température $T$ de la tache cathodique et de la densité de courant total qui la traverse. Nous avons localisé par des hachures le diagramme d'existence de l'arc.

Nous constatons que la zone d'existence de l'arc diminue lorsque le courant de décharge décroît jusqu'à une valeur minimale $I_{\mathrm{m}}$ pour laquelle l'échauffement de la cathode par effet Joule devient négligeable: $I_{\mathrm{m}} \simeq 30 \mathrm{~A}$ pour le potassium. Les travaux expérimentaux menés par ailleurs indiquent qu'au-dessus de cette limite le plasma d'arc a tendance à se stabiliser ce qui montrerait que la température du spot étant assez élevée, celui-ci émet des électrons par effet thermoionique.

On détermine aussi grâce à ce diagramme d'existence, l'intensité minimale par spot $I_{\mathrm{s}}$ en dessous de laquelle l'arc s'éteint. $I_{\mathrm{s}}=10 \mathrm{~mA}$ pour une cathode en potassium fondu.

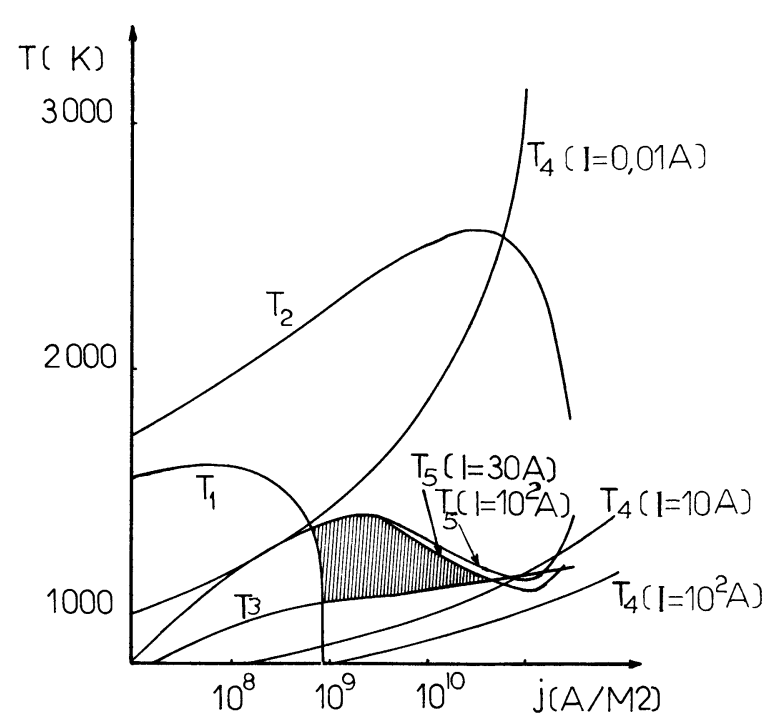

Fig. 5. - Diagramme d'existence de l'arc à cathode en potassium liquide.

Ni la figure 3, ni la figure 5 ne nous donnent de domaine d'existence aux fortes densités de courant $j$ pour les intensités de courant considérées ici $(0$ à $100 \mathrm{~A}$ ). La courbe $T_{5}$ tracée à partir de l'équation du bilan d'énergie (15) est limitée en température au point critique $T_{\mathrm{c}}$. Au-delà de $T_{\mathrm{c}}$ nous n'avons aucune information précise sur les variations des conductivités électrique et thermique des métaux liquides.

Lorsque nous comparons les figures 3 et 5 , nous constatons que l'allure des courbes déterminant le diagramme d'existence est la même dans les deux cas, cependant la zone d'existence est légèrement décalée vers des températures $T$ plus faibles lorsque l'on calcule le champ électrique à la cathode, non pas à l'aide de l'équation de Mac Keown, mais en utilisant le modèle de gaine défini par Ecker dans [2]. Ce modèle détaillé de la région cathodique montre que la densité ionique de charge d'espace $n_{+}$est très élevée. En effet, dans la zone quasi neutre $l_{n}$, nous devons avoir neutralité électrique globale :

$$
n_{+}=n_{-}+n_{\mathrm{e}} .
$$

Les électrons contre-diffusés imposent donc une forte densité de charge d'espace positive ce qui implique un champ électrique à la cathode également très élevé, donc une température de la tache cathodique plus faible, toutes choses égales par ailleurs.

Le tableau ci-contre indique quelques valeurs numériques des différents paramètres caractérisant la tache cathodique :

$$
\begin{array}{ccccccccc}
j\left(\mathrm{~A} / \mathrm{m}^{2}\right) & T(\mathrm{~K}) & j_{+}\left(\mathrm{A} / \mathrm{m}^{2}\right) & j_{\mathrm{e}}\left(\mathrm{A} / \mathrm{m}^{2}\right) & U_{\mathrm{c}}(\mathrm{V}) & E_{\mathrm{c}}(\mathrm{V} / \mathrm{m}) & T_{-}(\mathrm{K}) & n_{+}^{0}\left(\mathrm{~cm}^{-3}\right)=n_{-} & l_{\mathrm{sp}}(\mathrm{m}) \\
- & - & - & - & - & - & - & - & - \\
1,5 \times 10^{9} & 1250 & 6,18 \times 10^{8} & 8,82 \times 10^{8} & 6,8 & 8,35 \times 10^{8} & 11320 & 1,73 \times 10^{19} & 1,17 \times 10^{-8} \\
2,5 \times 10^{9} & 1200 & 6,62 \times 10^{8} & 1,82 \times 10^{9} & 10,7 & 9,77 \times 10^{8} & 19210 & 1,89 \times 10^{19} & 1,55 \times 10^{-8} \\
2,5 \times 10^{9} & 1350 & 6,78 \times 10^{8} & 1,84 \times 10^{9} & 6,0 & 8,44 \times 10^{8} & 9815 & 1,83 \times 10^{19} & 1,03 \times 10^{-8}
\end{array}
$$


En outre la figure 4 indique que la densité de courant ionique $j_{+}$à la cathode est faible. Sensiblement 25 à $30 \%$ du courant est transporté par les ions. Cette proportion peut atteindre $40 \%$ pour les densités de courant plus faibles $\left(1,5 \times 10^{9} \mathrm{~A} / \mathrm{m}^{2}\right)$ et une température de $1250 \mathrm{~K}$ par exemple.

Les résultats numériques, et plus particulièrement les valeurs élevées des densités $n_{+}^{0}$ et $n_{-}$et de la température $T$, obtenus à l'aide de ce modèle de gaine cathodique, justifient à posteriori l'hypothèse de l'E. T. L. dans la zone d'ionisation.

Si nous considérons que la chute de tension d'arc est essentiellement déterminée par la chute cathodique, la valeur de $U_{\mathrm{c}}$ calculée ici est en très bon accord avec les résultats expérimentaux.

Les limites des différentes régions d'émission définies par la relation (2) ont été représentées sur la figure 6 pour des métaux ayant un faible travail de sortie $\varphi$ tels que les métaux alcalins. Si nous portons les valeurs de $E_{\mathrm{c}}$ et $T$ calculées par la théorie sur cette figure, nous constatons que nous nous trouvons dans la région d'émission thermoionique. Cependant pour un point du diagramme d'existence correspondant à une densité de courant élevée, l'émission électronique à la cathode peut être une émission par effet de champ. Nous n'avons pas tenu compte non plus du facteur de rugosité qui a pour effet d'augmenter localement le champ électrique à la surface d'un métal liquide [11].

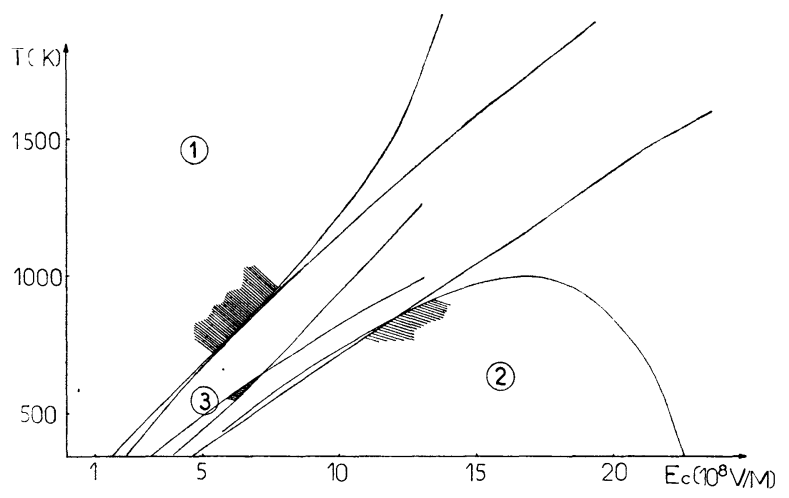

Fig. 6. - Limites des zones d'émission cathodique pour $\varphi=2 \mathrm{eV}$. (1) Région d'émission thermoionique ; (2) Région d'émission par effet de champ; (3) Région intermédiaire.

4. Conclusions. - L'intérêt de cette théorie est qu'elle permet de déterminer le diagramme d'existence de l'arc sans avoir recours à une grandeur mesurée expérimentalement comme c'est le cas lorsqu'on utilise l'équation de Mac Keown pour laquelle il faut connaître la valeur de la chute cathodique $U_{\text {c }}$. Le seul paramètre ajustable est l'intensité $I$ du courant de décharge.

Cette théorie distingue les électrons émis par la cathode des électrons thermalisés contre-diffusés. La densité des premiers joue un rôle négligeable, la densité des seconds influe fortement sur la valeur du champ électrique $E_{\mathrm{c}}$ à la cathode. Un développement de ce modèle devrait nous préciser davantage le mode d'émission cathodique. Certains aspects de mécanisme d'émission électronique peuvent être profondément modifiés par la présence d'un film d'oxyde à la surface de la cathode [12].

Nous avons résolu les équations pour $v=1$; il serait intéressant d'étudier l'évolution du diagramme d'existence de l'arc en considérant des ions plusieurs fois ionisés, car il est fort probable que de tels ions existent dans la région cathodique d'un arc électrique à cathode liquide.

Nous avons calculé les paramètres $\left(U_{\mathrm{d}}, T_{-}, n_{+}, T, j\right)$ caractérisant la tache cathodique sur du potassium liquide à l'aide du diagramme d'existence. Cependant beaucoup d'inconnues et d'imprécisions subsistent. Il serait notamment intéressant de retracer les zones d'existence en ayant au préalable fait une étude théorique des conductivités thermique et électrique des métaux liquides dans les conditions de pression et de température inhabituelles régnant dans la tache cathodique.

En écrivant le bilan d'énergie, nous nous sommes placés dans le cas des arcs avec spot. Si nous voulions considérer le cas des arcs sans spot observés par W. Weizel et W. Thouret [13], il faudrait tenir compte de la perte d'énergie de la cathode par conduction de la chaleur à travers le gaz neutre d'argon.

Le calcul montre que le champ électrique au point $x=0$, est, à un ordre de grandeur près, égal au champ cathodique. Mais si $\psi_{0}^{\prime}$ est inférieur à $\psi_{\text {ocr, }}^{\prime}$ nous ne pouvons plus considérer que la distribution des électrons rétrodiffusés est maxwellienne.

Nous n'avons pas tenu compte dans cette étude du mouvement de la tache cathodique à la surface du liquide. G. Ecker [14] a montré que seule l'équation de bilan d'énergie est légèrement affectée par ce déplacement.

\section{Bibliographie}

[1] ECKER, G., General Electric Report no 71-C-195, 1971.

[2] ECKER, G., Z. Naturforsch. 28a (1973) 3/4.

[3] MurPhy, E. L. et Good, R. H., Phys. Rev. 102 (1956) 1464.

[4] March, N. H., Liquid Metals (Pergamon Press) 1968.

[5] Daniel, E. et Vosko, S. H., Phys. Rev. 120 (1960) 2041.

[6] Tonks, L. et Langmuir, I., Phys. Rev. 34 (1929) 876.

[7] ECKER, G., Erg. d. exakten Naturwiss. 33 (1961) 1.

[8] Rich, J. A., J. Appl. Phys. 32 (1961) 1023.
[9] Grosse, A. V., Rev. Hautes Tempér. et Réfract. 3 (1966) 115.

[10] Kelley, Nouveau Traité de chimie minérale. Potassium (Masson et Cie Editeurs) 1963, tome II.

[11] Tonks, L., Phys. Rev. 48 (1935) 562.

[12] Guile, A. E. et Hitchсоск, A. H., J. Phys. D : Appl. Phys. 8 (1975) 663

[13] Weizel, W. et Thouret, W., Z. Phys. 131 (1952) 170.

[14] ECKeR, G., General Electric Report no 73 CRDO56-1973. 